\title{
In-house dining for phage
}

Although it has been proposed for some time that predation by lytic phage could have an important influence on the dynamics of Vibrio cholerae infection during epidemics, direct evidence to support this proposal has been lacking. Andrew Camilli and colleagues now provide the first evidence that phage predation drives genomic diversification of $V$. cholerae in vivo.

To gain insight into the impact of phage predation in the context of human bacterial infections, the authors used a plaque assay to detect phages in stool samples collected from cholera patients in Haiti in

2013. They identifed one sample with a high titre of a phage that was identified as a V. choleraespecific ICP2 phage and found that most single colony isolates of

$V$. cholerae from this sample were phageresistant.
Whole-genome sequencing of phage-resistant and -sensitive isolates showed that they were isogenic, except for mutations in the gene that encodes the major outer membrane porin OmpU (which the authors postulate functions as the ICP2 receptor), and further sequence analysis identified a total of six different ompU mutations. Similarly, genomic comparison of ICP2 phageresistant and -sensitive single colony isolates of $V$. cholerae from a stool sample obtained in Bangladesh in 2011 revealed that they were isogenic except for the presence of multiple phage resistance mutations in a single gene - toxR - which encodes a transcriptional activator for OmpU. In both cases, the presence of distinct mutations within a single host indicates that phage resistance was selected for on multiple, independent occasions.

What is the effect of the ICP2 resistance mutations on $V$. cholerae fitness and virulence during infection? Consistent with expression analysis, which showed that the ompU mutations did not affect OmpU expression levels, the authors found that these mutations had a negligible effect on $V$. cholerae fitness in vitro. However, in the infant rabbit infection model, the presence of ICP2 led to strong enrichment for $V$. cholerae isolates with an ompU mutation over wild-type $V$. cholerae. By contrast, the authors found that $V$. cholerae strains that contained any of the tox $R$ resistance mutations had highly reduced levels of OmpU and were avirulent in the infant rabbit model.

Together, this work provides the first direct evidence for phage predation of $V$. cholerae in vivo and demonstrates that such predation can drive genomic diversification and lead to altered virulence, and possibly altered transmissibility, of $V$. cholerae. Sheilagh Molloy

ORIGINAL RESEARCH PAPER Seed, K. D. et al. Evolutionary consequences of intra-patient phage predation on microbial populations. eLife 3 , e03497 (2014) 